\title{
Physiological Responses of General vs. Specific Aerobic Endurance Exercises in Soccer
}

\author{
Hassane Zouhal $^{\text {*1ABCDEFG }}$, PhD; Emmeran LeMoal ${ }^{1 \mathrm{ABCDEG}}$, PhD; Del P. Wong ${ }^{2 \mathrm{CEG}}$, PhD; Omar BenOunis ${ }^{\text {3BDG }}$, PhD; \\ Carlo Castagna $^{4 \mathrm{CEG}}$, PhD; Corentin Duluc ${ }^{\text {1BCFG }}$; Adam L. Owen ${ }^{\text {5BG }}$, PhD; Barry Drust ${ }^{6 \mathrm{ACDEG}}$, PhD
}

\author{
Authors' Affiliation: \\ 1. Movement, Sport and health Sciences \\ Laboratory (M2S), Rennes 2 University - \\ ENS Cachan, France \\ 2. Human Performance Laboratory, \\ Technological and Higher Education Institute \\ of Hong Kong, Hong Kong \\ 3. High Institute of Sport and Physical \\ Education, Ksar-Saïd, Manouba University, \\ Tunisia \\ 4. School of Sport and Exercise Sciences, \\ University of Rome Tor Vergata, Rome, Italy \\ 5. Rangers Football Club, Sports Science \\ Department, Glasgow, Scotland, UK \\ 6. Research Institute for Sport and Exercise \\ Sciences, School of Human Sciences, \\ Liverpool John Moores University, \\ Liverpool, UK

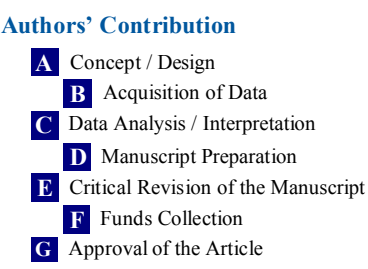 \\ * Corresponding Author; \\ Address: Movement, Sport and health \\ Sciences Laboratory (M2S), UFR-APS, \\ Rennes 2 University, Avenue Charles Tillon, \\ CS 24414, 35044 Rennes Cedex, France \\ E-mail: hassane.zouhal@univ-rennes2.fr \\ Received: Oct 20,2012 \\ Accepted: $\operatorname{Apr} 19,2013$ \\ Available Online: May 06, 2013
}

\begin{abstract}
Purpose: The study aimed to compare the physiological and perceptual responses of two high intensity intermittent aerobic exercises (HIIE), i.e. the 15s/15s exercise and an exercise on the Hoff track (HTE).

Methods: In this within-subject repeated measures study, seven high-level soccer players (Age: $24.1 \pm 4.5 \mathrm{yr}$; Height: $175 \pm 0.04 \mathrm{~cm}$; Body mass: $67.9 \pm 9.0 \mathrm{~kg}$; \% Body fat: $14.2 \pm 2.4 \%$ ) performed the two exercises with same total duration (25 minutes) in a randomized order: 1) a 15s/15s protocol at $120 \%$ of maximal aerobic speed (MAS), and 2) HTE. Heart rate (HR) and oxygen uptake $\left(\mathrm{VO}_{2}\right)$ were measured continuously throughout both exercises. The rating of perceived exertion (RPE) was measured 15 min after the end of each exercise. Blood lactate concentration ([La]) was measured at rest before each exercise, between and at the end of each set.
\end{abstract}

Results: The mean $\mathrm{VO}_{2}$ during $\mathrm{HTE}$ was significantly higher than $15 \mathrm{~s} / 15 \mathrm{~s}$ exercise $\left(39.3 \pm 2.3\right.$ vs. $36.8 \pm 1.9 \mathrm{~mL} / \mathrm{min} / \mathrm{kg}, P<0.05$. The total $O_{2}$ consumed was significantly higher $(P<0.05)$ during HTE $(66.8 \pm 7.6 \mathrm{~L})$ than during the $15 \mathrm{~s} / 15 \mathrm{~s}$ $(62.3 \pm 8.6 \mathrm{~L})$. Blood lactate [La] after the first set of HTE was significantly higher than the $15 \mathrm{~s} / 15 \mathrm{~s}(12.5 \pm 2.0$ vs. $10.6 \pm 2.0 \mathrm{mmol} / \mathrm{L}, P<0.05)$. However, RPE provided by players suggested that the $15 \mathrm{~s} / 15 \mathrm{~s}$ was more intense than the HTE $(13 \pm 1.8 v$ s. $11.7 \pm 1.4, P<0.05)$.

Conclusion: Our results demonstrate that $\mathrm{VO}_{2}$ and [La] were higher during HTE than during the 15s/15s when matched with duration. However, HTE was perceived less intense than $15 \mathrm{~s} / 15 \mathrm{~s}$. Thus, the use of HTE appears as an effective alternative for fitness coaches to develop aerobic endurance in soccer players.

Key Words: Football; Interval-Training; Aerobic Fitness; Rating of Perceived Exertion

\section{INTRODUCTION}

$\mathrm{S}^{\mathrm{o}}$ occer is the most popular sport in the world ${ }^{[1]}$. The performance in soccer depends on the technical, tactical, psychological and physical components. This sport requires well-developed physical fitness to be successfully played ${ }^{[2]}$. During competitive soccer match-play, elite players cover a distance of 10-12 km ${ }^{[1,3-5]}$ at an average intensity close to the anaerobic lactate threshold, being $80-90 \%$ of maximal heart rate or $70-80 \%$ of maximal oxygen uptake $\left(\mathrm{VO}_{2 \max }\right)^{[6]}$. During a game, it is estimated that the aerobic metabolism provides $90 \%$ of the total energy expenditure ${ }^{[1,7]}$. The information from time-motion and physiological analysis shows the importance of the aerobic fitness in soccer.

It is well established that the aerobic endurance capacity is closely related to soccer performance. In 1980, Smaros ${ }^{[8]}$ has reported a significant correlation between $\mathrm{VO}_{2 \max }$ and the total distance covered during match play. Moreover, $\mathrm{VO}_{2 \max }$ also affects the number of sprints attempted during a match ${ }^{[9]}$. Furthermore, the importance of $\mathrm{VO}_{2 \max }$ has been reflected by a rank correlation of the most successful teams in the Hungarian $1^{\text {st }}$ division championship ${ }^{[10]}$. In addition, Wislöff et al ${ }^{[11]}$ have reported an aerobic power- 
success relationship by demonstrating a significant difference between top team and lower team in Norwegian $1^{\text {st }}$ division championship.

In soccer training, many strategies to improve $\mathrm{VO}_{2 \max }$ have been developed. The repetition of high intensity intermittent aerobic exercises (HIIE) in-line (at maximal aerobic speed or supra-maximal aerobic speed) is one of the most widely practiced training methods ${ }^{[12,13]}$. However, many soccer coaches and fitness coaches believe that this kind of exercise is not specific to soccer because there is no soccer technical component.

Other forms of soccer-specific aerobic training have been designed and identified as a similar physiological stimulus to high intensity running such as small-sided games ${ }^{[14,15]}$ or a specifically designed soccer track where the player dribbles a soccer ball instead of regular running ${ }^{[16,17]}$. The soccer specific track designed by Hoff et al ${ }^{[16]}$ allows simulating actions of soccer such as dribbling and changing direction with the ball. This kind of exercise improves the technical skills and also physical fitness of soccer players representing a gain of time during the training. During the HTE, players have to dribble the ball through the routed obstacles ${ }^{[16,18]}$, with established distance of 290 $\mathrm{m}$ for one lap.

The use of the Hoff track, which allows the measure of $\mathrm{VO}_{2}$ during soccer-specific exercise ${ }^{[19]}$, may be as effective as aerobic interval training because of similar heart rate responses to a small-sided game ${ }^{[16,18]}$. Two studies have investigated the effects of training on the Hoff track on endurance capacity of young soccer players: Chamari et al ${ }^{[18]}$ have demonstrated that in addition of regular training, $4 \times 4$ minutes of dribbling the ball around the track and $4 \times 4$ minutes of smallsided games improved $\mathrm{VO}_{2 \max }$ for $12 \%$. In addition, a 10 weeks study of McMillan et al ${ }^{[17]}$ have showed that performing high intensity 4 x 4 minutes intervals dribbling a soccer ball around this track together with regular soccer training is effective enough to improve $\mathrm{VO}_{2 \max }$ for $9.4 \%$ in elite young soccer players with no negative interference with strength, jumping ability and sprint performance. However, no control groups were investigated in these two studies, which did not allow isolating the influence of training on the Hoff track in the gains of $\mathrm{VO}_{2 \max }$ observed.

It could be useful to determine the physiological and perceptual solicitations of an HIIE on the Hoff track and to compare it to a traditional model of high intensity aerobic running exercise frequently used in soccer. In training-related studies, increased $\mathrm{VO}_{2 \max }$ of well-trained subjects is generally observed after training at high intensity i.e. $90-100 \% \mathrm{VO}_{2 \max }{ }^{[20-23]}$. Therefore, it can be assumed that the percentage of $\mathrm{VO}_{2 \max }$ attained and the time for which it is sustained could serve as a good criterion to judge the effectiveness of the stimulus ${ }^{[24]}$.

Up to now in this topic it is known that Hoff track and HIIE are accompanied by an increase of $\mathrm{VO}_{2 \max }$ in soccer players. However, it is still unknown which one is more effective. Thus, the purpose of this study was to compare the physiological and perceptual solicitations of two HIIE: $15 \mathrm{~s} / 15 \mathrm{~s}$ at $120 \%$ of maximal aerobic speed (MAS) and an integrated exercise on the Hoff track (HTE). As during the Hoff track there is several accelerations, decelerations, turning and jumping, we hypothesized that the physiological (e.g. $\mathrm{VO}_{2}$ and blood lactate concentration) and perceptual solicitations (Rating of Perceived Exertion (RPE) will be higher during this kind of exercise than the classical HIIE $15 \mathrm{~s} / 15 \mathrm{~s}$ at $120 \%$ MAS.

\section{METHODS AND SUBJECTS}

\section{Participants:}

Seven high-level male soccer players (National level in France) volunteered to participate in this study (Age: $24.1 \pm 4.5 \mathrm{yr}$; Height: $175 \pm 0.04 \mathrm{~cm}$; Body mass: $67.9 \pm$ $9.0 \mathrm{~kg}$; \% Body fat: $14.2 \pm 2.4 \%$ ). Prior to participation, the players underwent a medical examination and were fully informed about the experimental procedures and the risk and benefit associated with this research design. All participants signed the written consent prior to the data collection. Players were aware that they could withdraw from the study at anytime without associated penalties.

\section{Protocol:}

In this study, we compared the oxygen consumption, blood lactate concentrations and rating of perceived exertion between two high intensity intermittent 
aerobic exercises: $15 \mathrm{~s} / 15 \mathrm{~s}$ at $120 \%$ of maximal aerobic speed (MAS) and an integrated exercise on the HTE in highly trained soccer players. All players performed three running sessions between April and May on the same outdoor synthetic pitch, separated by 3-7 days. To minimize any effects of diurnal variation, the three testing sessions were conducted within $2 \mathrm{~h}$ of the same time of the day. During the first session, the peak oxygen uptake $\left(\mathrm{VO}_{2 \text { peak }}\right)$, the peak heart rate $\left(\mathrm{HR}_{\text {peak }}\right)$ and maximal aerobic speed (MAS) for each player were measured. In the second and third sessions, $15 \mathrm{~s} / 15 \mathrm{~s}$ at $120 \%$ of MAS $(15 \mathrm{~s} / 15 \mathrm{~s})$ and HTE were performed randomly. The total duration (25 minutes) was the same for the two exercises. This experiment was conducted during the competitive period and there was only one match per week. Players played the match on Sunday and each test was performed on Wednesday or Thursday.

\section{Anthropometry and physiological measures:}

Anthropometrical characteristics of all players were determined in laboratory conditions before each session. Body mass was measured to the nearest $0.1 \mathrm{~kg}$ with a digital scale (OHAUS, Florhman Park, NJ). Height was measured with a standing stadiometer and recorded with a precision of $1 \mathrm{~mm}$. A recently calibrated Harpenden calliper (Holtain, Cross-well, UK) was used to estimate body fat percentage (\%Fat) from four-skinfold thicknesses according to the method of Durnin and Rahaman $\mathrm{V}^{[25]}$. Fat Free Mass (FFM) was calculated by subtracting total body fat from total body mass.

For each test session, oxygen uptake $\left(\mathrm{VO}_{2}\right)$ was recorded continuously by means of a gas exchange telemetric system $\left(\mathrm{VO}_{2000}\right.$, Medical Graphics, Minnesota, USA). The system is lightweight ( $740 \mathrm{~g})$ with the main sample unit attached to the back and a battery pack on the chest to minimize the disruption of range of motion during movement. This system has been previously validated using a cart system in our laboratory and by other authors ${ }^{[26,27]}$. Before each test, this system was calibrated using ambient air and two precision reference gases of known concentrations. Data were averaged every $5 \mathrm{~s}$ for subsequent analysis. During the course of the experiment, the receiving unit was positioned beside the running track in the outdoor stadium. Throughout the exercise, heart rate (HR) was measured and recorded every $5 \mathrm{~s}$ with a heart rate monitor (Sport Tester S 610i, Polar, Kempele, Finland) for each player.

\section{Maximal graded test:}

Each player performed an incremental running Vam Eval test ${ }^{[28]}$ to determine $\mathrm{HR}_{\text {peak }}$ and MAS. The initial speed was $8.5 \mathrm{~km} \cdot \mathrm{h}^{-1}$ and increased by $0.5 \mathrm{~km} . \mathrm{h}^{-1}$ every minute. During this test, the pace was controlled by sounds emitted through a computer software program to ensure precise control of speed by setting an audible cadence. Each subject was encouraged to exert a maximum effort. The test was stopped when the player could not maintain the required speed. The mean value in $\mathrm{VO}_{2}$ during the last elapsed minute at this stage was recorded. Achievement was accepted when players fulfilled at least three of the four following criteria: a plateau in $\mathrm{VO}_{2}$ despite an increase in running speed, a respiratory exchange ratio higher than 1.10 , a maximal HR near the age-predicted maximal HR (220-age in year), a blood lactate concentration higher than 8.0 mmol. $\mathrm{L}^{-1}$ and the apparent exhaustion of the subjects. MAS $\left(\mathrm{km} \cdot \mathrm{h}^{-1}\right)$ was then defined as the lowest running speed completed during the incremental exercise protocol.

During the second and third sessions, the player engaged in a standardized warm-up consisting of 15min jogging at a low speed, 10-15 min of stretching and three to five 20 to $30 \mathrm{~m}$ "run-throughs" at increasing speeds. Following stretching, the heart rate monitor and the metabolic harness were placed on the player. At the start line, the participant was given the standard starting commands, at which point they approached the start line and they began the time-trial.

\section{Intermittent exercise in line (15s/15s):}

The subjects had to run 4 series of 4 minutes $(15 \mathrm{~s}$ in line at an intensity of $120 \%$ of individual MAS interspersed by $15 \mathrm{~s}$ of passive recovery). Working intensity was at $90-95 \%$ of each player's $\mathrm{HR}_{\max }$, and 3 min of jogging at $70 \%$ of $\mathrm{HR}_{\max }$ were allowed between series.

\section{Hoff track (HTE):}

For this test the subjects had to run 4 bouts of 4 minute work periods dribbling the ball around the designated track (Fig. 1). The player had to reach and maintain a 


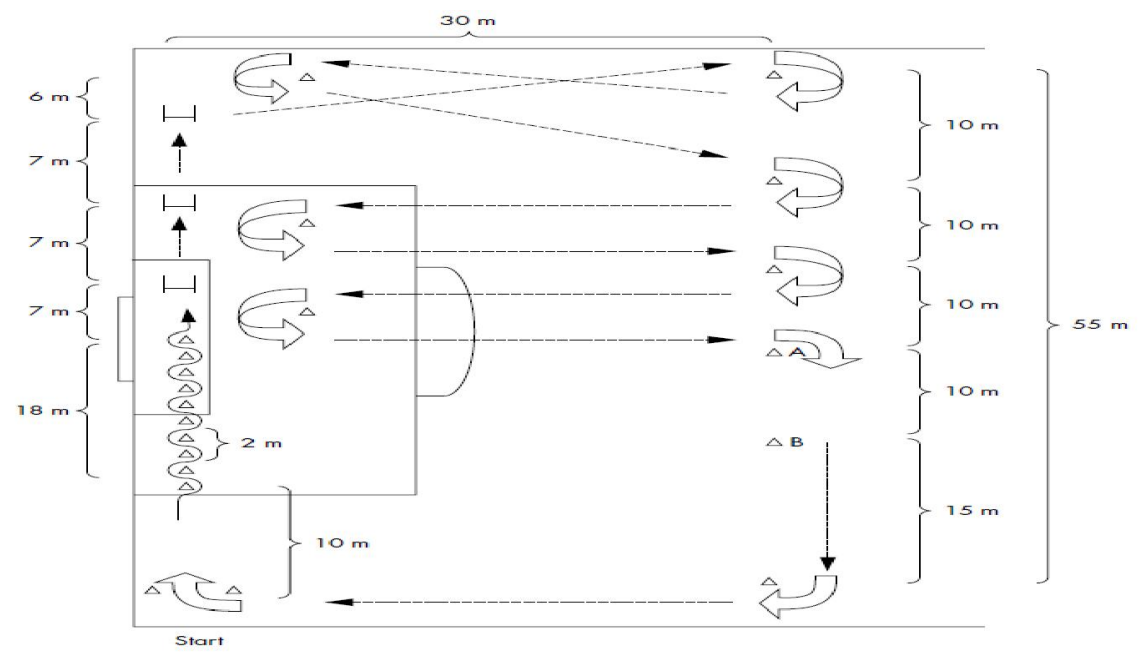

Fig. 1: The soccer specific track adapted from Höff et al. (2002)

heart rate (HR) range from 90 to $95 \%$ of $\mathrm{HR}_{\max }$, with work periods separated by $3 \mathrm{~min}$ of jogging at $70 \%$ of $\mathrm{HR}_{\max }$. This test was adapted from McMillan et al ${ }^{[17]}$.

\section{Blood lactate measurements:}

For the measurement of blood lactate concentrations (Microzym-L analyzer, SGI, Toulouse, France), capillary blood samples were obtained from the player's finger in $25 \mu \mathrm{l}$ heparinised capillary tubes at rest before each test, 3 minutes after the end of the Vam Eval test, at the end of the warm-up of each intermittent exercise (HTE and 15s/15s) and between each set and five minutes after these two exercises.

\section{Rating of Perceived Exertion (RPE):}

Finally, the subject accomplished the RPE based on Borg's scale (20-point scale) 15 minutes after the end of the two exercises. The use of RPE is considered as a good indicator of internal load of soccer ${ }^{[29]}$.

\section{Statistical Analyses:}

Data are reported as mean \pm SD. After testing for normal distribution (Kolmogorov-Smirnov test), data were analysed using ANOVA with repeated-measures and the Newman-Keul's post-hoc test was used in the event of significance.

The effect size (ES) was calculated to assess meaningfulness of differences. Effect sizes of $>0.8$, $0.8-0.5,<0.5-0.2$, and $<0.2$ were considered as large, moderate, small and trivial respectively [30]. Relationships between parameters were assessed using Pearson's product-moment correlation. The magnitude of the correlations was determined using the modified scale: trivial: $\mathrm{r}<0.1$; low: $0.1-0.3$; moderate: $0.3-0.5$; high: $0.5-0.7$; very high: $0.7-0.9$; nearly perfect $>0.9$; and perfect: $1^{[31]}$.

$\mathrm{P}<0.05$ was accepted as statistical significance. Statistical analyses were carried out with the SigmaStat 1.0 program (Jandel Scientific, San Rafael, US).

\section{RESULTS}

The maximal values determined during the maximal graded test of the players are showed in table 1 .

Physiological parameters, blood lactate and RPE scores of the players determined during the $15 \mathrm{~s} / 15 \mathrm{~s}$ test and HTE are presented in Table 2. As shown in Table 2 , there were no significant differences between the $15 \mathrm{~s} / 15 \mathrm{~s}$ exercise and HTE concerning peak HR, mean HR and the precent of peak HR of the players. In the same way, there were no significant differences concerning heart rate kinetics during the four sets of the two intermittent exercises, the HTE and the $15 \mathrm{~s} / 15 \mathrm{~s}$ (Fig. 2). The relative $\mathrm{VO}_{2}$ was significantly higher during HTE than during the $15 \mathrm{~s} / 15 \mathrm{~s}$ exercise $(P<0.05)$. 
Table 1: Physiological parameters determined at rest and during the maximal graded exercise testing $(n=7)$

\begin{tabular}{|c|c|c|c|c|c|}
\hline $\begin{array}{c}\text { HRrest } \\
\text { (beats. } \mathrm{min}^{-1} \text { ) }\end{array}$ & $\begin{array}{c}\text { HR peak } \\
\text { (beats.min } \text { min }^{-1}\end{array}$ & $\underset{\left(\mathrm{mmol.}^{\left.-\mathrm{L}^{-1}\right)}\right.}{\left[\mathrm{La}_{0}\right]_{\mathrm{b}}}$ & $\underset{\left(\text { mmol. }^{-1}\right)}{\left[\mathrm{La}_{\max }\right]_{\mathrm{b}}}$ & $\begin{array}{c}\mathrm{VO}_{2 \max } \\
\left(\mathrm{mL} \cdot \mathrm{min}^{-1} \cdot \mathrm{kg}^{-1}\right)\end{array}$ & $\begin{array}{c}\text { MAS } \\
\left(\mathrm{km} \cdot \mathrm{h}^{-1}\right)\end{array}$ \\
\hline 63(16) & $195(8)$ & $2.2(0.4)$ & $13.9(4.1)$ & $58(6)$ & $17.1(0.6)$ \\
\hline
\end{tabular}

HR: heart rate. MAS: maximal aerobic speed. $\left[\mathrm{La}_{0}\right]_{\mathrm{b}}$ : lactate concentration measured at rest. [La $\left.\mathrm{max}\right]_{\mathrm{b}}$ : lactate concentration measured 3 minutes after the end of the exercise

When recovery periods were excluded, the $\mathrm{VO}_{2}$ and HR of the players during the $15 \mathrm{~s} / 15 \mathrm{~s}$ exercise were around $46 \mathrm{~mL} / \mathrm{min} / \mathrm{kg}$ and 171 beats $/ \mathrm{min}$ respectively which correspond to $80 \%$ of $\mathrm{VO}_{2 \text { peak }}$ and $88 \%$ of $\mathrm{HR}_{\text {peak. For }} \mathrm{HTE}$ these values were around 50 $\mathrm{mL} / \mathrm{min} / \mathrm{kg} \quad(P<0.05)$ and 173 beats $/ \mathrm{min}$, which correspond to $87 \%$ of $\mathrm{VO}_{2 \text { peak }}(P<0.05)$ and $89 \%$ of $\mathrm{HR}_{\text {peak. Total oxygen consumption in liter was }}$ significantly higher during the two first sets of the HTE compared to the $15 \mathrm{~s} / 15 \mathrm{~s}$ (Fig. 3).

Blood lactate concentrations increased significantly in response to intermittent exercises and there was a significant difference between the values determined after the first set of the $15 \mathrm{~s} / 15 \mathrm{~s}$ and HTE for all the players $(p<0.05)$ (Table 2$)$. Hence, as shown by the RPE score values, players perceived HTE more difficult than the $15 \mathrm{~s} / 15 \mathrm{~s}$ exercise $(\mathrm{p}<0.05)$.

Nearly perfect correlation was observed between the total $\mathrm{O}_{2}$ consumed during $\mathrm{HTE}$ and the $15 \mathrm{~s} / 15 \mathrm{~s}$ exercise $(\mathrm{r}=0.97 ; P<0.05)$.

\section{DISCUSSION}

The main purpose of this study was to compare the physiological and perception responses of two HIIE: $15 \mathrm{~s} / 15 \mathrm{~s}$ at $120 \%$ of MAS and an integrated exercise on the Hoff track (HTE). Our results demonstrated that oxygen consumption and blood lactate concentrations compared to the $15 \mathrm{~s} / 15 \mathrm{~s}$. Surprisingly, they perceived HTE less intense than the $15 \mathrm{~s} / 15 \mathrm{~s}$ test. Since it has been demonstrated that the amount of oxygen consumed during exercise could serve as a good criterion to judge the effectiveness of this exercise ${ }^{[24]}$, the HTE appears to be more effective to increase aerobic fitness ${ }^{[17]}$. Hence it may provide more variety during training, combining physical and technical components.

From the methodological point of view, the choice to compare these two exercises was based on the training methods frequently used by fitness soccer coaches to develop aerobic endurance fitness of

Table 2: Physiological parameters, blood lactate concentrations and RPE score determined during the $15 \mathrm{~s} / 15 \mathrm{~s}$ and the HTE for all the players $(n=7)$

\begin{tabular}{|c|c|c|c|}
\hline Variable & Variables $(n=7)$ & $15 s / 15 s$ & HTE \\
\hline \multirow{3}{*}{ Heart Rate } & Peak heart rate (beats/min) & $187(8)$ & $184(9)$ \\
\hline & Mean heart rate (beats/min) & $156(9)$ & $156(11)$ \\
\hline & Peak heart rate $(\%)$ & $69.7(3.3)$ & $69.9(5.1)$ \\
\hline \multirow{3}{*}{$\mathrm{VO}_{2}$ peak } & Absolute $\mathrm{VO}_{2}$ peak (L/min) & $3.7(0.5)$ & $3.9(0.4)$ \\
\hline & Relative $\mathrm{VO}_{2}$ peak $(\mathrm{mL} / \mathrm{min} / \mathrm{kg})$ & $53.3(3.32)$ & $57.5(3.2)$ \\
\hline & Relative $\mathrm{VO}_{2}$ peak (mL/min/kgFFM) & $62.5(4.2)$ & $68.0(4.5)$ \\
\hline \multirow{3}{*}{ Mean $\mathrm{VO}_{2}$} & Absolute $\mathrm{VO}_{2}$ peak $(\mathrm{L} / \mathrm{min})$ & $2.49(0.34)$ & $2.67(0.30)^{*}$ \\
\hline & Relative $\mathrm{VO}_{2}$ peak $(\mathrm{mL} / \mathrm{min} / \mathrm{kg})$ & $36.8(1.9)$ & $39.3(2.3)^{*}$ \\
\hline & Relative $\mathrm{VO}_{2}$ peak (mL/min/kgFFM) & $42.2(2.6)$ & $45.9(2.9)^{*}$ \\
\hline \multicolumn{2}{|l|}{ Total $\mathrm{VO}_{2}(\mathrm{~L})$} & $62.3(8.6)$ & $66.8(7.6)^{*}$ \\
\hline \multirow{5}{*}{ Blood Lactate (mmol/L) } & Warm-up & $4.6(1.7)$ & $5.1(1.5)$ \\
\hline & Set 1 & $10.6(2.0)$ & $12.5(2.0)^{*}$ \\
\hline & Set 2 & $11.1(1.9)$ & $11.5(1.9)$ \\
\hline & Set 3 & $12.1(3.8)$ & $11.9(2.7)$ \\
\hline & End of exercise & $8.4(2.6)$ & $8.4(2.3)$ \\
\hline \multicolumn{2}{|c|}{ Rating of Perceived Exertion Score } & $13(1.8)$ & $11.7(1.4)^{*}$ \\
\hline
\end{tabular}




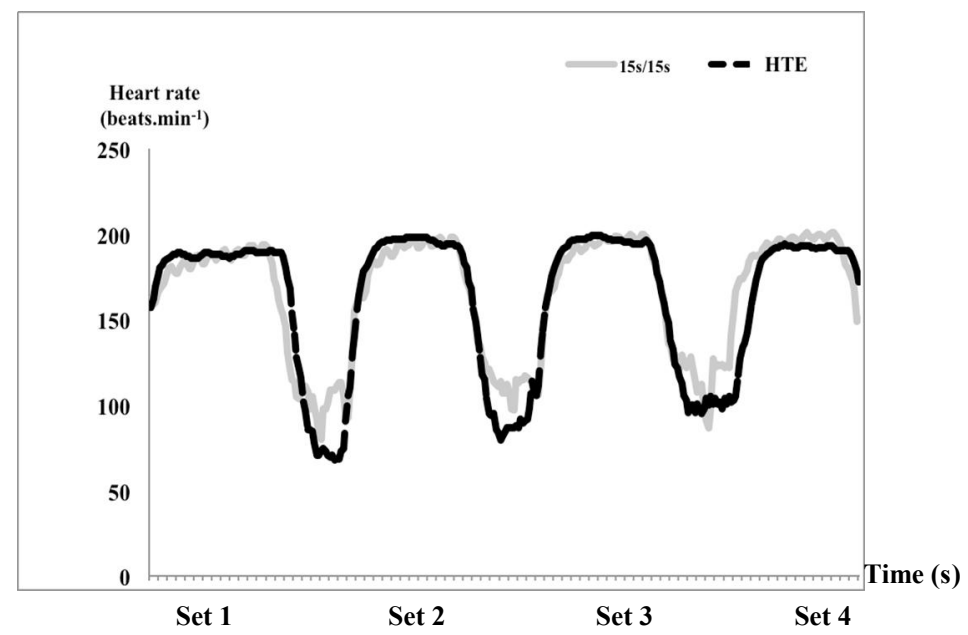

Fig. 2: Heart rate kinetics during the fourth sets of the two intermittent exercises, the HTE and the $15 \mathrm{~s} / 15 \mathrm{~s}(\mathrm{n}=7)$

players. Hence, the choice was also based on the scientific data of the literature. In fact, it was demonstrated that the $15 \mathrm{~s} / 15 \mathrm{~s}$ exercise induces a significant increase of $\mathrm{VO}_{2 \max }{ }^{[32]}$. Moreover, this type of exercise is more comparable to the efforts realized in soccer than continuous exercises or longer interval exercises.

During HTE, the values of HR attained by our players are comparable to previous studies using the same exercise ${ }^{[16,17]}$. These values indicated that this type of exercise induces higher solicitation of cardiovascular system. These measures are confirmed by the higher values of $\mathrm{VO}_{2}$ determined during HTE which were around $87 \% \mathrm{VO}_{2 \text { peak }}$. In their study, Hoff et al ${ }^{[16]}$ observed that this exercise was accomplished at $95.5 \% \mathrm{HR}_{\max }$ and $91.7 \% \mathrm{VO}_{2 \max }$.

We hypothesized that the $\mathrm{VO}_{2}$ will be higher during HTE than during the $15 \mathrm{~s} / 15 \mathrm{~s}$ exercise. Our results support this hypothesis that the total amount of $\mathrm{VO}_{2}$ was significantly higher during HTE than during the $15 \mathrm{~s} / 15 \mathrm{~s}$ exercise despite similar HR values. These results can mainly be explained by the nature of the exercises. In fact, during the integrated exercise on the Hoff track, there are several accelerations, decelerations, turning and jumping which induced higher solicitation of muscular mass than the $15 \mathrm{~s} / 15 \mathrm{~s}$ exercise and therefore a higher $\mathrm{VO}_{2}$. However, these higher values of $\mathrm{VO}_{2}$ were only observed during the

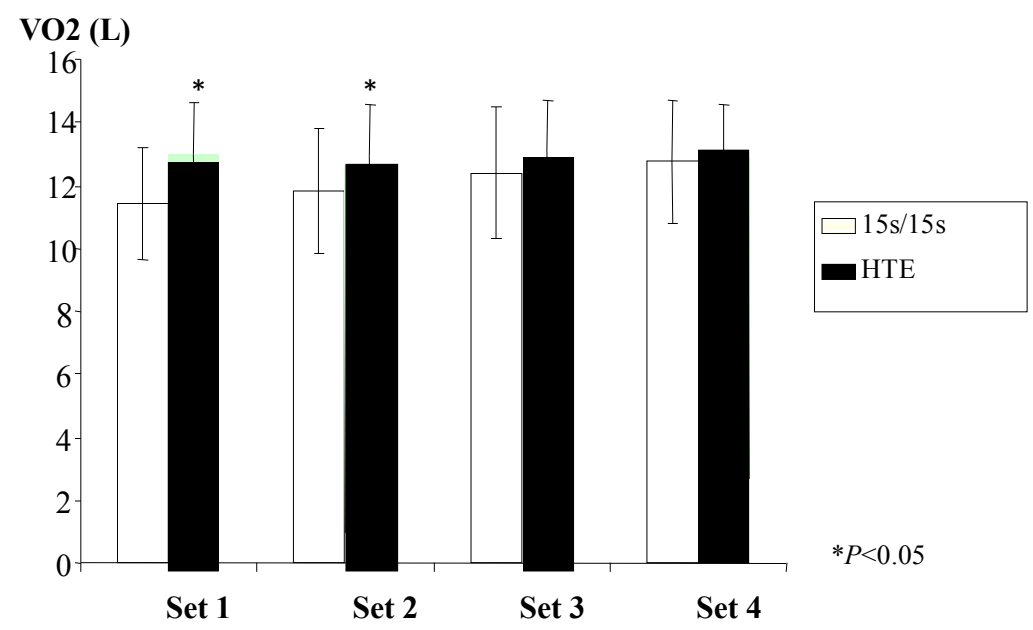

Fig. 3: Total oxygen consumption in liter for the four sets of the two intermittent exercises, the HTE and the $15 \mathrm{~s} / 15 \mathrm{~s}(\mathrm{n}=7)$ 
first two sets of the exercises. These results can be explained by the duration of each set chosen in our study (e.g. $4 \mathrm{~min}$ ). This duration is too short for the $15 \mathrm{~s} / 15 \mathrm{~s}$ exercise to significantly increase $\mathrm{VO}_{2}$ at the beginning of the exercise and longer duration may be needed ${ }^{[24]}$. Consequently, our results showed that the physiological solicitation is higher during HTE than during the $15 \mathrm{~s} / 15 \mathrm{~s}$ exercise and particularly during the first two sets. This observation is confirmed by the blood lactate concentration, which was significantly higher only after the first set of HTE. This can be explained by the characteristics of this exercise in which more muscular mass is engaged and may be because more energy is provided by anaerobic pathway during the first set.

The players of our study perceived the $15 \mathrm{~s} / 15 \mathrm{~s}$ exercise more difficult than HTE as indicated by the higher value of the RPE. This result is surprising since the players consume more $\mathrm{O}_{2}$ and produce more lactate during HTE than during the $15 \mathrm{~s} / 15 \mathrm{~s}$ exercise. Therefore, this result can be explained mainly by the fact that the integrated exercise as HTE is not perceived by the players as a physical exercise and may diminish a fatigue sensation.

Moreover, according to Hoff and Helgerud [13], ideally, endurance training for soccer players should be carried out using the ball, which may develop technical skills and keep the motivation at high level.

Our results demonstrate that oxygen consumption and blood lactate concentrations were higher during the Hoff track than during the $15 \mathrm{~s} / 15 \mathrm{~s}$ (especially during the first set) in highly trained soccer players. Despite that, players perceived the Hoff track less difficult than $15 \mathrm{~s} / 15 \mathrm{~s}$ test. Thus, the use of the Hoff track for aerobic training appears as an interesting alternative to develop endurance in soccer players. However, fitness coaches have had to propose this Hoff track far from the soccer game because it may induce more anaerobic participation to the energy production. Moreover, this type of integrated exercise might raise different problems in terms of the subjects and coaches' control of intensity, control of the ball etc. that might influence training execution and response.

Limitations, practical applications and future research: The major limitation of our study was the small number of participants (only 7 players), which did not allow us to be more affirmative. In addition, the small number of players did not allow us to examine the physiological responses of the $15 \mathrm{~s} / 15 \mathrm{~s}$ and HTE according to playing positions.

The information provided by this study results support the already reported notion of training prescription specificity for endurance. Soccer strength and conditioning coaches should elaborate different programs and sessions to increase aerobic fitness of soccer players. They can use high aerobic intermittent exercises such as $15 \mathrm{~s} / 15 \mathrm{~s}$, integrated exercise such as the Hoff track or small-side games.

Further research is needed in this topic. For example, conducting a longitudinal study in high- level soccer players to investigate which kind of exercise is more effective in improving endurance fitness. Hence, it will be interesting to investigate the level of recovery after these types of training exercises and the mechanisms involved. This will allow us to program this type of training with more accuracy and pertinence.

\section{CONCLUSION}

Our study demonstrated clearly that the integrated exercise such as HTE induces higher physiological solicitation than do the $15 \mathrm{~s} / 15 \mathrm{~s}$ exercise. Thus, higher physiological load could be obtained by using this type of a specifically designed soccer track where the subjects dribble the soccer ball instead of regular running. Moreover, the players perceive this exercise as less intense.

\section{ACKNOWLEDGMENTS}

The authors would like to thank all the players for their coach's participation. The study protocol was conducted in accordance with the Code of Ethics of the World Medical Association (Declaration of Helsinki) and with the guidelines of the Ethical Committee of Rennes 2, which had approved the experimental protocol, and the procedures involved.

Conflict of interests: None 


\section{REFERENCES}

[1] Bangsbo J. The physiology of soccer--with special reference to intense intermittent exercise. Acta Physiol Scand Suppl 1994;619:1-155.

[2] Stolen T, Chamari, K, Castagna C, et al. Physiology of soccer: an update. Sports Med 2005;35:501-36.

[3] Mohr M, Krustrup P, Bangsbo J. Match performance of high-standard soccer players with special reference to development of fatigue. $J$ Sports Sci 2003;21:519-28.

[4] Di Salvo V, Baron R, Tschan H, et al. Performance characteristics according to playing position in elite soccer. Int J Sports Med 2007; 28:222-7.

[5] Vigne G, Gaudino C, Rogowski I, et al. Activity profile in elite italian soccer team. Int J Sports Med 2010;31:304-10.

[6] Helgerud J, Engen LC, Wisloff U, et al. Aerobic endurance training improves soccer performance. Med Sci Sports Exerc 2001;33:192531.

[7] Iaia FM, Rampinini E, Bangsbo J. High intensity training in football. Int J Sport Physiol Perform 2009;4:291-306.

[8] Smaros G. Energy usage during a football match. In: Vecciet L (ed). Proceedings of the $1^{\text {st }}$ International Congress on Sports Medicine Applied to Football. Rome: D Guanillo, 1980.

[9] Rampinini E, Sassi A, Morelli A, et al. Repeated-sprint ability in professional and amateur soccer players. Appl Physiol Nutr Metab 2009;34:1048-54.

[10] Apor P. Successful formulae for fitness training. In: Reilly T, Lees A, Davis K, et al (eds). Science and Football London: E \& FN Spon. 1988 .

[11] Wisloff U, Helgerud J, Hoff J. Strength and endurance of elite soccer players. Med Sci Sports Exerc 1998;30:462-7.

[12] Dupont G, Millet GP, Guinhouya C, et al. Relationship between oxygen uptake kinetics and performance in repeated running sprints. Eur J Appl Physiol 2005;95:27-34.

[13] Hoff J, Helgerud J. Endurance and strength training for soccer players: physiological considerations. Sports Med 2004;34:165-80.

[14] Dellal A, Chamari K, Pintus A, et al. Heart rate responses during small-sided games and short intermittent running training in elite soccer players: a comparative study. J Strength Cond Res 2008;22:1449-57.

[15] Hill-Haas, SV, Dawson B, Impellizzeri FM, et al. Physiology of small-sided games training in football: a systematic review. Sports Med 2011;41:199-220.

[16] Hoff J, Wisloff U, Engen LC, et al. Soccer specific aerobic endurance training. Br J Sports Med 2002;36:218-21.

[17] McMillan K, Helgerud J, Macdonald, et al. Physiological adaptations to soccer specific endurance training in professional youth soccer players. Br J Sports Med 2005;39:273-7.

[18] Chamari K, Hachana Y, Kaouech F, et al. Endurance training and testing with the ball in young elite soccer players. Br J Sports Med 2005;39:24-8.

[19] Kemi OJ, Hoff J, Engen LC, et al. Soccer specific testing of maximal oxygen uptake. J Sports Med Phys Fitness 2003;43:139-44.

[20] Wenger HA, Bell GJ. The interactions of intensity, frequency and duration of exercise training in altering cardiorespiratory fitness. Sports Med 1986;3:346-56.

[21] Robinson DM, Robinson SM, Hume PA, et al. Training intensity of elite male distance runners. Med Sci Sports Exerc 1991;23:107882 .

[22] Tabata I, Nishimura K, Kouzaki M, et al.. Effects of moderate-intensity endurance and high-intensity intermittent training on anaerobic capacity and $\mathrm{VO}_{2}$ max. Med Sci Sports Exerc 1996;28:1327-30.

[23] Jones AM, Carter H. The effect of endurance training on parameters of aerobic fitness. Sports Med 2000;29:373-86.

[24] Thevenet D, Tardieu M, Zouhal H, et al. Influence of exercise intensity on time spent at high percentage of maximal oxygen uptake during an intermittent session in young endurance-trained athletes. Eur J Appl Physiol 2007;102:19-26.

[25] Durnin JV, and Rahaman MM. The assessment of the amount of fat in the human body from measurements of skinfold thickness. Br $J$ Nutr 1967;21:681-9.

[26] Olson TP, Tracy JE, Dengel DR. Validity of a low flow Pneumotach and Portable Metabolic System for measurement of basal metabolic rate. Med Sci Sports Exerc 2003;35:143.

[27] Kautza BC, Kastello GM, Sothmann MS. Validation of MedGraphics $\mathrm{VO}_{2000}$ Portable Metabolic Analyzer and a modified Pneumotachometer. Med Sci Sports Exerc 2004;36:s222.

[28] Cazorla G, and Léger L. Comment évaluer et développer vos capacités aérobies. Epreuves de course navette et épreuve Vameval. Eds AREAPS, Bordeaux, 1993.

[29] Impellizzeri FM, Rampinini E, Coutts AJ, et al. Use of RPE-based training load in soccer. Med Sci Sports Exerc 2004;36:1042-7.

[30] Cohen J. Statistical Power Analysis for the Behavioral Sciences. $2^{\text {nd }}$ ed. Hillsdale, NJ: Lawrence Erlbaum Associates. 1988.

[31] Hopkins WG. A scale of magnitudes for effect statistics. Available at: http://www.sportsci.org/resource/stats/index.html. Access date: Dec 2009.

[32] Helgerud J, Hoydal K, Wang E, et al. Aerobic high-intensity intervals improve $\mathrm{VO}_{2 \max }$ more than moderate training. Med Sci Sports Exer 2007;39:665-71. 